\title{
Study of the Growth Morphology of AZO at the Substrate-Film Interface
}

Tara Dhakal, Abhishek Nandur, Rachel Christian, Daniel VanHart, and Charles R Westgate

Center for Autonomous Solar Power (CASP), Binghamton University, Binghamton, NY 13902

Aluminum doped zinc oxide (AZO) thin film is gaining momentum as a transparent conducting oxide (TCO) because its optical and electrical properties are optimal for a photovoltaic device [1]. We report a growth of AZO thin film using an Atomic Layer Deposition (ALD) system, which is known to deposit layers on a substrate with atomic precision [2]. The precursors used for aluminum and zinc oxides were Trimethylaluminum (TMA) and Dimethylzinc (DMZ) respectively. Alternative cycles of TMA and DMZ were introduced to the heated deposition chamber such that a desired aluminum doping was obtained on the AZO films grown. The films were grown on 4" diameter single crystalline $\mathrm{Si}(100)$ and float-glass substrates heated at $325^{\circ} \mathrm{C}$ in nitrogen ambient. On silicon, a buffer layer of $\mathrm{Al}_{2} \mathrm{O}_{3}$ is also grown before the $\mathrm{AZO}$ layer. An optimal aluminum doping on the AZO film of thickness $575 \mathrm{~nm}$ gave a sheet resistance $\left(\mathrm{R}_{\mathrm{s}}\right)$ of $100 \Omega / \square$, which after 30 min utes annealing at $400^{\circ} \mathrm{C}$ in an argon environment, reduced to $25 \Omega / \square$ with corresponding resistivity of 1.4 x $10^{-3}$ S.cm (Table 1). The carrier concentration and hall mobility were $2.39 \times 10^{20} \mathrm{~cm}^{-3}$ and 17.76 $\mathrm{cm}^{2} \mathrm{~V}^{-1} \mathrm{~s}^{-1}$ respectively. Optical measurement showed $90 \%$ transmission in most of the visible spectrum.

Surface morphology of the thin films was imaged by Scanning Electron Microscopy (SEM) [3]. The as-grown films are relatively smooth with uniform distribution of grains which are around 60-100 $\mathrm{nm}$ long and 10-20 nm wide as shown in Figure 1. The stoichiometry of the film was estimated by Energy Dispersive Spectrometry (EDS) analysis, which showed that aluminum doping was around 3 atomic percent. The X-ray Diffraction spectra of the film showed all characteristic ZnO hexagonal lattice with space group P63mc (186). The film texture is dominantly (100) oriented. Roughness analysis was performed on the images taken by atomic force microscopy (AFM) on as-grown and annealed samples as shown in Figure 2. The annealed sample surface was found to be relatively smoother as compared to the as-grown films. The growth morphology at the interface was examined using a JEOL JEM-2100F field emission transmission electron microscope (TEM). HRTEM images across the interface are taken to characterize the growth orientation. Nanobeam electron diffraction (NBED) technique is used to further verify the crystal structure.

In conclusion, AZO films with very smooth surface and uniform texture are successfully grown by ALD system and the micro-structure is studied using imaging techniques such as SEM, AFM and TEM. This transparent conducting oxide has a potential to replace high cost TCOs such as tin-doped indium oxide (ITO).

References

[1] Tadatsugu Minami, Semicond. Sci. Technol. 20 (2005) S35

[2] S. M. George, A. W. Ott, and J. W. Klaus , J. Phys. Chem. 100 (1996) 13121

[3] J.I. Goldstein et al., Scanning Electron Microscopy and X-ray Microanalysis, Plenum, New York, 1992.

[4] This work was supported in part by Defense Advanced Research Projects Agency (DARPA) award number HR0011101002. 
TABLE 1. Electrical Characterization of the AZO film using Hall Effect measurement

\begin{tabular}{lccccc}
\hline $\begin{array}{l}\text { Anneal } \\
\text { Time }(\min .)\end{array}$ & $\mathrm{R}_{\mathrm{s}}(\Omega / \square)$ & $\rho(\Omega . \mathrm{cm})$ & $\mathrm{n}\left(\mathrm{cm}^{-3}\right)$ & $\mu\left(\mathrm{cm}^{2} \mathrm{~V}^{-1} \mathrm{~s}^{-1}\right)$ & Type \\
\hline 0 & 97.77 & $5.623 \mathrm{E}-03$ & $1.86 \mathrm{E}+20$ & 6.51 & n-type \\
5 & 32.67 & $1.879 \mathrm{E}-03$ & $2.56 \mathrm{E}+20$ & 13.18 & n-type \\
30 & 25.95 & $1.492 \mathrm{E}-03$ & $2.39 \mathrm{E}+20$ & 17.76 & n-type \\
60 & 32.67 & $1.775 \mathrm{E}-03$ & $2.62 \mathrm{E}+20$ & 13.53 & n-type
\end{tabular}

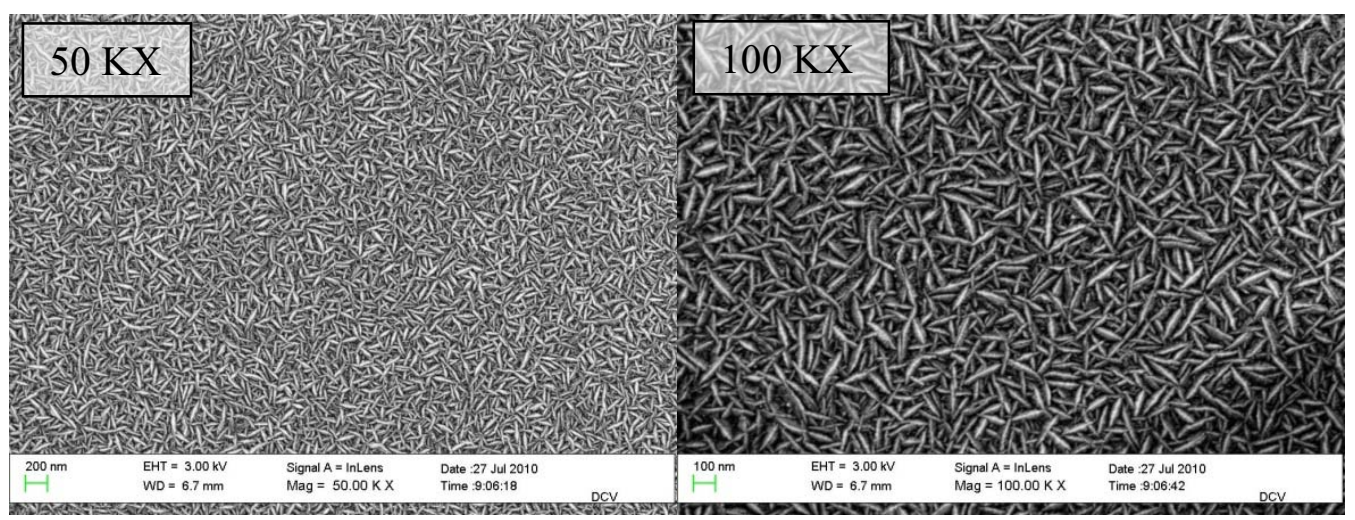

FIG. 1. SEM images of AZO thin film grown by ALD system.

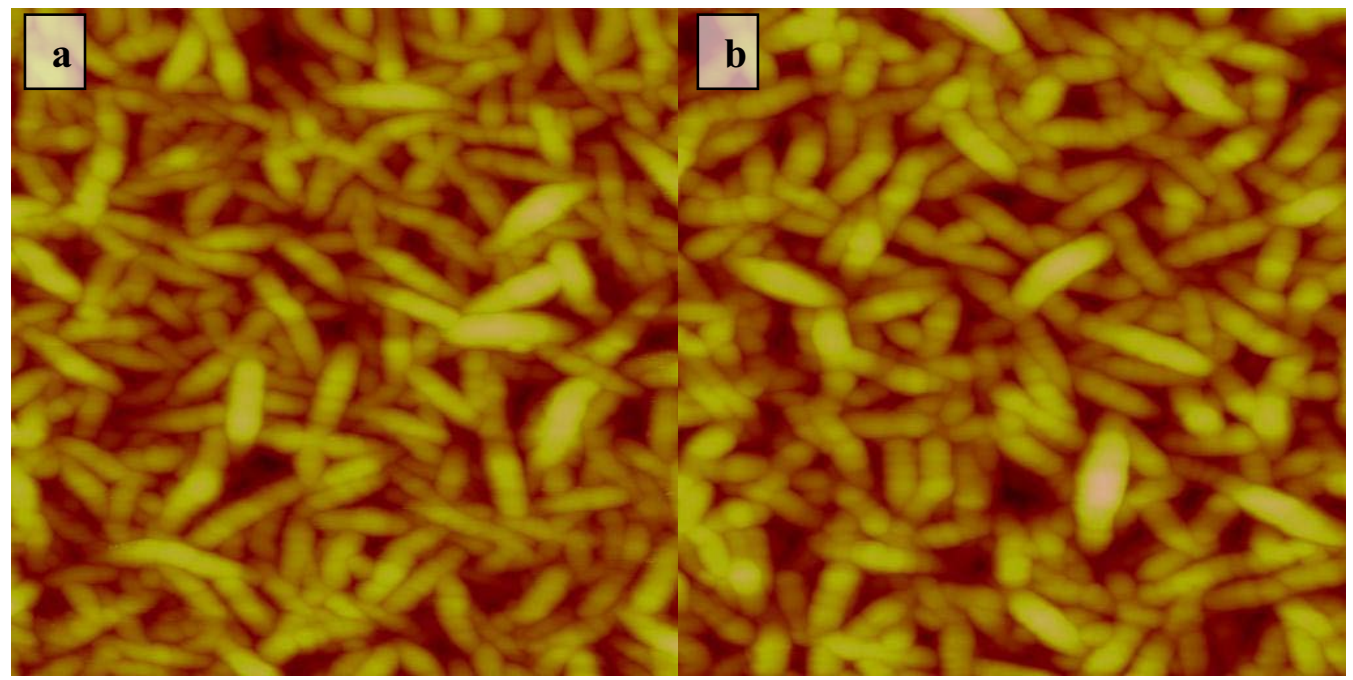

FIG. 2. AFM images $(1 \mu \mathrm{m} \times 1 \mu \mathrm{m})$. (a) As-grown film. (b) Annealed film at $400^{\circ} \mathrm{C}$ in argon ambient for $30 \mathrm{~min}$. The roughness $\left(\mathrm{R}_{\mathrm{q}}\right)$ of the as-grown film was $7.31 \mathrm{~nm}$, which improved to $6.72 \mathrm{~nm}$ for annealed film. 